This manuscript is the last draft of the original article published as : Peigneux, P., S. Laureys, S. Fuchs,

X. Delbeuck, C. Degueldre, J. Aerts, G. Delfiore, A. Luxen and P. Maquet (2001). "Generation of rapid eye movements during paradoxical sleep in humans." Neuroimage 14(3): 701-8.

\title{
Generation of Rapid Eye Movements during Paradoxical SleEp in Humans
}

TO APPEAR IN: NEUROIMAGE

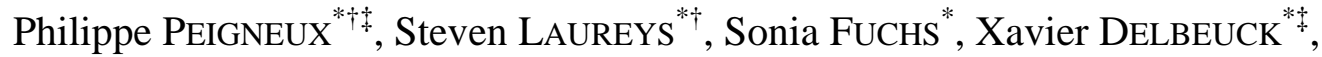 \\ Christian Degueldre ${ }^{*}$, Joel Aerts ${ }^{*}$, Guy Delfiore $^{*}$, André LuXeN $^{*}$ and Pierre Maquet ${ }^{*} \dagger$ \\ * Cyclotron Research Center, University of Liège, Belgium \\ ${ }^{\dagger}$ Department of Neurology, CHU Sart Tilman, University of Liège, Belgium \\ $\ddagger$ Department of Neuropsychology, University of Liège, Belgium
}

Corresponding author: Pierre MAQUET

Cyclotron Research Center, University of Liège (ULg)

Bât. B30, Sart Tilman

B-4000 Liège

BELGIUM

Phone : +32 (4) $3663687 \quad$ Fax : +32 (4) 3662946

E-mail: maquet@pet.crc.ulg.ac.be 


\section{ABSTRACT}

Although rapid eye movements (REMs) are a prominent feature of paradoxical sleep (PS), their origin and functional significance remain poorly understood in humans. In animals, including non human Primates, REMs during PS are closely related to the occurrence of the so-called PGO waves, i.e., prominent phasic activities recorded throughout the brain but predominantly and the most easily in the pons $(\mathrm{P})$, the lateral geniculate bodies $(\mathrm{G})$ and the occipital cortex $(\mathrm{O})$. Therefore, and because the evolution of species is parsimonious, a plausible hypothesis would be that during PS in humans, REMs are likewise generated by mechanisms similar to PGO waves. Using positron emission tomography (PET) and iterative cerebral blood flow measurements by $\mathrm{H}_{2}{ }^{15} \mathrm{O}$ infusions, we predicted that the brain regions where the PGO waves are the most easily recorded in animals would be differentially more active in PS than in wakefulness, in relation with the density of the REMs production [i.e., we looked for the condition (PS versus wakefulness) by performance (REMs density) interaction]. Accordingly, we found a significant interaction effect in the right geniculate body and in the primary occipital cortex. The result supports the hypothesis of the existence of processes similar to PGO waves in humans, responsible for REMs generation. The interest of the presence of PGO waves in humans is outstanding because the cellular processes involved in, or triggered by, PGO waves might favor brain plasticity during PS.

KEY WORDS : ponto-geniculo-occipital waves, PGO, paradoxical sleep, rapid eye movements, REMs, PET, sleep 


\section{INTRODUCTION}

In humans, the origin and functional significance of rapid eye movements (REMs) generation during paradoxical sleep (PS) remain poorly understood. In animals however, including in non human Primates (Vuillon-Cacciuttolo and Seri 1978), it is known that spontaneous visual activity during PS is generated in the ponto-mesencephalic reticular formation and closely relates to the occurrence of the so-called ponto-geniculo-occipital (PGO) waves (Mouret et al., 1963). PGO waves are prominent phasic bioelectrical potentials which occur in isolation or in bursts during the transition from slow wave sleep (SWS) to PS or during PS it-self [for reviews see (Callaway et al., 1987; Datta 1999a)]; they constitute one of the distinguishing features of PS. Although observed from many parts of the animal brain (Hobson 1964), they are most easily recorded in the pons (Jouvet et al., 1959), the lateral geniculate bodies (Mikiten et al., 1961) and the occipital cortex (Mouret et al., 1963), hence their name. Among various functions, PGO waves are hypothesized to promote brain development and to facilitate brain plasticity (Datta 1999a). Hence, PGO waves seem to represent a fundamental process of PS in animals.

Several observations suggest that PGO waves also occur during human sleep. In epileptic patients, direct intracerebral recordings in the striate cortex showed monophasic or diphasic potentials during PS, isolated or in bursts (Salzarulo et al., 1975). The finding was corroborated in normal subjects by surface electroencephalography (EEG) showing transient occipital and/or parietal potentials time-locked to REMs (McCarley et al., 1983). Finally, source dipoles of magnetoencephalography (MEG) signal were localized in the brainstem, thalamus, hippocampus and occipital cortex during PS (Inoué et al., 1999). Therefore, and because the evolution of species is parsimonious, a plausible hypothesis would be that REMs during PS are generated in humans by mechanisms similar or identical to PGO waves in animals. 
Obvious ethical and technical reasons preclude the use of electrophysiological intracerebral techniques to prove the existence of PGO waves in humans. Nevertheless, positron emission tomography (PET) and cerebral blood flow (CBF) determination make it possible to unravel indirectly their existence through their haemodynamic consequences. In neuroanatomical terms, it follows from the hypothesis of presence of PGO waves in humans that the neural activity of the brain regions from which PGO are the most easily recorded in animals (i.e. the dorsal meso-pontine tegmentum, the lateral geniculate bodies and the occipital cortex) should be more tightly related to spontaneous ocular movements during PS, than during wakefulness. Here, we tested this prediction using PET, looking for those cerebral areas where local neuronal activity correlated more to the REMs density during PS than during wakefulness.

\section{MATERIAL AND METHODS}

The analysis is based on 72 scans (44 scans in PS and 28 during wakefulness) collected in 12 subjects in the framework of a PET study of human sleep described elsewhere (Maquet et al., 2000). Only information relevant to the present topic is presented here.

\section{Population and experimental protocol}

Twelve young (mean $22.9 \pm 3.5$ years), healthy, right-handed male volunteers gave their written informed consent to participate in this study approved by the Ethical Commission of the Faculty of Medicine of the University of Liège. Each subject spent three consecutive nights in the PET scanner, during their usual sleep time. Polysomnography monitoring during the first 2 nights allowed to check for any abnormality in sleep (insomnia, sleep fragmentation, PS onsets...) and accustomed participants to the experimental setting. They were selected for the third night if they could maintain 20 minutes of continuous stage II sleep, SWS and PS on both habituation nights. During the third night, 12 PET scans were performed both during various stages of sleep when polysomnography showed steady 
characteristic sleep patterns, and during waking at rest with eyes closed in complete darkness. At least 2 waking, 2 stage II sleep, 2 SWS and 3 PS scans were obtained in all subjects. During waking scans, the subjects had to stay still, eyes closed. No instruction was provided concerning eye position, in order to record spontaneous waking, endogenous, saccades.

\section{$\underline{\text { Polysomnography acquisition and ocular movement counts }}$}

Polysomnography was recorded with a Synamp (Neuroscan, NeuroSoft Inc., Sterling, VA), at $500 \mathrm{~Hz}$, with a bandwidth 0.15 to $100 \mathrm{~Hz}$ and $\mathrm{A}_{1}$ as reference. For the first two nights, only $\mathrm{C} 3$ and $\mathrm{C} 4$ channels were recorded. For the third night, twenty-eight scalp electrodes were placed according to the 10-20 system. In all cases, vertical and horizontal electro-oculograms, chin electromyogram and electrocardiogram were recorded on bipolar montages.

Polysomnographic recordings were scored using standard criteria (Rechtschaffen and Kales 1968). In addition to the presence of REMs, PS was always easily recognized by the lowvoltage rapid activity on EEG recording as well as by muscular atonia and twitches. To facilitate the comparison with the data from a previous PET study targeting the relationship between ocular movements and metabolism during sleep and wake states, (Hong et al., 1995), the same method for counting REMs was used. All discernible horizontal sharp-onset REMs were counted in both directions, irrespective of their amplitude, during the 90s-duration of each scan (during PS and wakefulness). One of the authors (PM) recounted a sample of records after 3 months. Intra-observer scoring reliability was excellent (Pearson correlation coefficient $r=0.90$ ). These raw counts of REMs were used as covariate of interest in the statistical analysis of the PET data.

\section{$\underline{\text { PET and MRI acquisitions }}$}

PET data were acquired on a Siemens CTI 951 R 16/31 scanner in 3D mode. Throughout the night, the subject's head was stabilized by a thermoplastic facemask secured to the head holder (Truscan imaging, MA), and a venous catheter was secured in a left antebrachial vein. 
First, a 20-min transmission scan was acquired for attenuation correction using three rotating sources of $68 \mathrm{Ge}$. Then, regional cerebral blood flow, taken as a marker of local neuronal activity (Jueptner and Weiller 1995), was estimated during twelve emission scans. Each scan consisted of 2 frames: a 30-second background frame and a 90-second acquisition frame. The slow intravenous water $\left(\mathrm{H}_{2}{ }^{15} \mathrm{O}\right)$ infusion begun 10 seconds before the second frame. Six mCi (222 MBq) were injected for each scan, in 5 cc saline, over a period of 20 seconds. The infusion was totally automated in order not to disturb the subject during the scanning period. Data were reconstructed using a Hanning filter (cutoff frequency: 0.5 cycle/pixel) and corrected for attenuation and background activity. For each subject, structural T1-weighted magnetic resonance imaging (MRI) scans $(0.96 \times 0.96 \times 1.50 \mathrm{~mm}$ voxel size) were obtained on a 1.5T Magnetom scanner (Siemens, Erlangen, Germany).

\section{$\underline{\text { Brain imaging data analysis }}$}

Data were analyzed using statistical parametric mapping (SPM99; Welcome Department of Cognitive Neurology, Institute of Neurology, London, UK) implemented in MATLAB. For each subject, all scans were realigned together. A mean PET image was created, to which the subject's MRI scan was coregistered. Then, PET and MRI images were normalized to a standard PET template using the same transformations (Frackowiak et al., 1997). Finally, PET images were smoothed using a Gaussian kernel of $16 \mathrm{~mm}$ full width at half maximum.

The scan condition (PS or wakefulness), covariate (REMs counts), condition by covariate interaction and subject (block) effects were estimated at each voxel according to the general linear model. Global flow adjustment was performed by proportional scaling. Areas of significant changes were determined using linear contrasts of condition estimates. The resulting set of voxel values for each contrast constituted a map of the t statistic $[\operatorname{SPM}(\mathrm{T})]$, thresholded at $\mathrm{p} \leq 0.001(\mathrm{~T} \geq 3.14)$. When we had a priori hypotheses on the brain areas of interest, significance level was set at $\mathrm{p}$ (uncorrected) $\leq .001(\mathrm{~T} \geq 3.14)$. Correction for 
multiple comparisons were then performed using a small volume correction thresholded at $\mathrm{p}<$ .05 (in a spherical volume of $5 \mathrm{~mm}$ ). The volumes of interest were positioned on the average structural MRI. When no a priori localization was available, the results were considered significant at $\mathrm{p}<0.05$ at the voxel level, after correction for multiple comparisons in the whole brain volume.

The first contrast estimated the effect of ocular movements (raw counts) during wakefulness. Since the cerebral correlates of spontaneous saccades during wakefulness is not yet described precisely, in contrast to other voluntary (stimulus- or memory-guided) saccades (Leigh and Zee 1991), no a priori localization was used in the interpretation of the resulting SPM maps. For the effect of REMs density (counts) in PS, a priori loci of potential activations were predicted on the basis of what is known (a) from previous studies which have correlated REMs density to regional metabolism during PS (Hong et al., 1995), and (b) from REMs generation during PS in animals (Callaway et al., 1987): the pontine and mesencephalic tegmentum, the superior colliculi, the thalamus (lateral geniculate bodies, pulvinar and intralaminar thalamic nuclei), the hippocampus and the parahippocampal gyrus, the occipital cortex, the precuneus, the parietal cortex near the intraparietal sulcus (parietal eye field), the frontal eye field, the supplementary eye field, the dorso-lateral prefrontal cortex and the anterior cingulate cortex.

The third contrast of interest estimated the effect of the interaction between the condition (PS versus Wakefulness) and the covariate (REMs counts). A priori loci of differential activations were those from which PGO are the most easily recorded in animals (Callaway et al., 1987) : the dorsal meso-pontine tegmentum, the lateral geniculate bodies and the occipital cortex. 


\section{RESULTS}

\section{$\underline{\text { Rapid eye movements count }}$}

The mean number of REMs during the scan duration (90s) was $20.5 \pm 17.6($ mean \pm SD) during PS and $15.0 \pm 15.8$ during wakefulness. REMs counts did not differ significantly between conditions (Student $t$ test, $\mathrm{p}=.58$ ).

$\underline{\text { Effect of spontaneous ocular movements during wakefulness }}$

Regression with REMs count was mainly related to the activation of frontal regions. However, no result survived the $\mathrm{p}<0.05$ level after correction for multiple comparisons at the voxel level. In the absence of a priori predictions, results significant at $\mathrm{p}<0.001$ (uncorrected) are provided for completeness only (see table 1).

\section{Effect of REMs in PS}

During PS, REMs counts correlated significantly with rCBF in the mesencephalon and the thalamus, including the geniculate body, and, in the right hemisphere, in the parahippocampal gyrus, the striate cortex, the precuneus, the right anterior cingulate cortex and the supplementary motor area (see table 2).

\section{Condition (PS versus wakefulness) by REMs counts interaction}

A significant interaction was observed bilaterally in the striate cortex and in the right geniculate body (see table 3 and figure). This result indicates that, in these cerebral regions, regional $\mathrm{CBF}$ variations are significantly more correlated to REMs density during PS than during wakefulness. It should be stressed that the contralateral voxel, in the left geniculate body, was activated but did not reach the statistical threshold \{coordinates $[-16-24-4], \mathrm{T}=$ 2.66, $\mathrm{p}=0.006$ uncorrected $\}$. Although not hypothesized a priori, an activation was also detected in the right primary sensorimotor and premotor cortices, parahippocampal gyrus and left anterior cingulate cortex. These latter activations are reported for completeness. 


\section{DISCUSSION}

\section{$\underline{\text { Methodological considerations }}$}

In the present analysis, REMs were considered as identical during PS and wakefulness. Some authors did not observe any differences between these two types of ocular movements (Jeannerod and Mouret 1963), but others describe that REMs during PS are slower than in wakefulness, in monkeys (Fuchs and Ron 1968) as in man (Fukuda et al., 1981). Moreover, they are superimposed on slow rolling movements (Fuchs and Ron 1968), and at variance with wakefulness, there is no strict relationship between REMs amplitude and velocity during PS (Aserinsky et al., 1985). Such differences between ocular movements in PS and wakefulness may themselves suggest that their mechanisms of production are different. Analyses of the maximum velocity/amplitude relationships for spontaneous waking and paradoxical sleep saccades in the cat further support this hypothesis (Vanni-Mercier et al., 1994). It has been proposed that PGO waves and REMs during PS are two corollary outputs of a common generator structure, which allows a relative uncoupling between their respective discharges (Vanni-Mercier et al., 1998). In consequence, differences in REMs quality does not preclude the significance of our results. Likewise, in contrast to Hong et al. (1995), we did not provide any instruction concerning eye movements during waking scans in order to observe spontaneous saccades during both wakefulness and PS.

$\underline{\text { Functional segregation of REMs in PS - Comparison with literature }}$

Like previous related PET studies, we found a significant correlation between REMs counts and $\mathrm{rCBF}$ in the anterior cingulate cortex during PS (Hong et al., 1995). It is not surprising, since the region is known to be both activated during PS (Maquet et al., 1996) and involved in oculomotor control (Paus et al., 1993). Medial frontal cortex CBF was correlated to REMs counts both during PS and wakefulness in the study of Hong et al. (1995). We found that supplementary motor area CBF was correlated to REMs counts in PS, while CBF in more 
inferior parts of medial frontal cortex correlated to REMs counts during wakefulness. Although the medial aspect of premotor cortex has not been systematically explored during PS, recent PET data from our laboratory showed the premotor cortex more active in PS than during wakefulness (group 3 in Maquet et al., 2000). Medial premotor cortex is activated by the generation of ocular saccades (Petit et al., 1993) and putatively corresponds to the supplementary eye field described in monkeys (Schlag and Schlag-Rey 1987).

We did not observe any correlation in the dorso-lateral prefrontal cortex in PS, in line with the relative hypoactivity of associative prefrontal areas during PS (Maquet et al., 1996). However, contradictory results reported positive (Hong et al., 1995) or negative (Braun et al., 1998) associations between $\mathrm{rCBF}$ and REMs density in this structure. Also, our finding is at odd with the report of a positive correlation between metabolic rates for glucose (CMRGlu) and REMs density in a similar set of cortical areas during PS and wakefulness (Hong et al., 1995). Various causes may explain these discrepancies. Hong et al. (1995) and Braun et al. (1998) studies estimated the rCBF-REMs correlation during PS alone, while we aimed to identify regions which were differentially activated in relation to REMs counts in PS as compared to wakefulness. Also, Hong et al. (1995) used quantitative CMRGlu measurements while we qualitatively estimated regional CBF. Their subjects maintained PS only during the first 32 minutes of the uptake period, which cannot preclude that some waking metabolism influence their correlation. Our CBF measurements are much quicker (90 s) than previous studies (Hong et al., 1995; Braun et al., 1998), and both PET data and REMs counts included in the design matrix were obtained solely in PS, representing pure PS functional neuroanatomy.

\section{Differential functional segregation of REMs during PS and wakefulness}

REMs are more tightly related during PS than wakefulness to the regional cerebral activity in the primary occipital cortex and the right lateral geniculate body (LGB). These results are in 
good agreement with our hypothesis that mechanisms similar to PGO waves would occur in humans but they deserve some qualifications.

First, we did not observe any activation in the dorsal ponto-mesencephalic tegmentum, although, in animals, PGO waves are known to be generated in the peribrachial region. Part of the pons might have been missed because of the limited axial sampling of our scanner (10.8 $\mathrm{cm}$ ); field of view was centered on parietal, motor and premotor areas, near the vertex. Also, REMs-related neuronal population might be too small to be detected by PET CBF measurements.

Second, the activated thalamic area is centered on the right LGB, although bilateral activation is observed using a more liberal statistical threshold. Animal studies indicate that PGO waves may be lateralized and related to ipsiversive or contraversive REMs (Nelson et al., 1983). Because we did not discriminate rightward from leftward REMs, we cannot be sure that this asymmetric activation represents the predominance of one particular direction of REMs. More importantly, the activation does not seem to involve other thalamic nuclei implicated in the generation of voluntary saccades, mainly the pulvinar and the intralaminar nuclei (Leigh and Zee 1991). To the best of our knowledge, functional neuroimaging studies in humans did not report LGB activation in response to the generation of saccades during wakefulness. In consequence, REMs-related LGB activation seems specific to PS mechanisms and, from our hypothesis, would be related to an activity similar to PGO waves.

Third, our results did not fit with a popular hypothesis - the so-called "scanning hypothesis"which argues that ocular movements during PS reflect the oneiric activity of the dreamer, who is actually scanning the visual content of the dream (Dement 1964). In neuroanatomical terms, this hypothesis implies that REMs should be generated at the cortical level (Hong et al., 1997), following a top-down process similar to what is observed for the generation of voluntary saccades during wakefulness (Leigh and Zee 1991). Hong et al. (1995) study was 
thought to support the scanning hypothesis (Hong et al., 1997), because a set of brain areas was shown to be similarly correlated with REMs during PS and wakefulness. Yet, apart from the methodological considerations mentioned above, our results suggest that most of the spontaneous visual activity during REM sleep relies on the activity of brain regions well known to be involved in PGO waves generation. however, it might be that mechanisms similar to PGO waves in humans lead to an increase of the activity of the visual system during PS, which may in turn favor the visual vividness of the dreaming activity. Hence, scanning and PGO hypotheses are not mutually exclusive.

Lastly, the primary occipital cortex was more associated with REMs in PS than in wakefulness. Occipital cortex involvement in saccade generation remains controversial. On the one hand, during wakefulness, the occipital cortex seems activated mainly when saccades are generated in response to external visual stimuli (Bodis et al., 1997; Darby et al., 1996), but on the other hand, occipital CBF was shown to decrease in proportion of the saccade frequency during wakefulness (Paus et al., 1995). Visual mental imagery is a condition occurring during full-conscious wakefulness, where primary occipital cortex (Le Bihan et al., 1993) and even LGB (Chen et al., 1998) may be activated in the absence of external visual stimulation. However, cerebral regional activations during visual mental imagery have not been correlated, to our knowledge, to simultaneous REMs, although task-dependent eye movements may occur during visual imagery tasks (Brandt et al., 1997; Hodgson et al., 2000). Likewise, during PS, the subject does not receive any external visual stimulation: the occipital cortex has to be activated in relation to REMs by endogenous mechanisms. However, in contrast to mental imagery, the subject is not likely to be able to willingly produce mental images during PS (the particular case of lucid dreamers is not discussed here). We propose that endogenous mechanisms similar to PGO waves in animals are good candidates to explain this PS-related occipital activation. 


\section{$\underline{\text { Functional implications }}$}

The presence of PGO wave mechanisms in humans might have an important functional significance. In several mammal species, including non-human Primates, PGO waves seem to represent a fundamental process of PS, [for review see (Callaway et al., 1987)]. Yet, the nature of the information transferred by PGO waves from the peribrachial area to the rostral structures remains unknown. PGO waves have been implicated in various non exclusive processes such as intervention in dream generation (Hobson and McCarley 1977), alerting reaction to external stimuli or internal signals (Bowker and Morrison 1976), sensorimotor integration through the transmission of an efferent copy of ocular movements to the visual system (Callaway et al., 1987), or facilitation of brain plasticity (Datta 1999a). The latter hypothesis is supported by a body of arguments. First, in animals, PGO activity seems to play a significant role in central nervous system maturation: abolition of PGO waves in kittens leads to a maturational deficit in LGB (Davenne and Adrien 1984), additive to the maturational impairment following visual deprivation (Shaffery et al., 1999). Second, PGO waves might participate in learning and memory in adults. In humans, intensive learning periods are accompanied by increased REMs density in subsequent PS (Smith and Lapp 1991) and retention levels after learning a Morse code correlated with subsequent REMs frequency in PS (Mandai et al., 1989). Likewise, PGO wave density increases after aversive conditioning in rats (Datta 1999b). Third, several independent works point to the importance of PGO processes in the organization of cerebral networks during PS. Although particularly large in the occipital cortex, PGO waves appear widespread over the cortex and occur synchronously across the cortical mantle. They synchronize high frequency $(20-50 \mathrm{~Hz})$ activities when induced by brainstem stimulation (Amzica and Steriade 1996). These fast oscillations involve thalamic and widespread cortical areas (Llinas and Ribary 1993) and are presumed to play a substantial role in cognitive functions during wakefulness (Llinas et al., 
1998). Fast oscillations recorded during sleep, including in PS, are modified by previous instrumental conditioning during wakefulness (Amzica et al., 1997). Hence, PGO activities during PS might synchronize fast oscillations that would convey experience-dependent information in thalamo-cortical and intra-cortical circuits.

\section{CONCLUSION}

The definitive proof for the existence of PGO waves in humans would necessitate in-situ electrophysiological recordings that specify their site of origin, demonstrate their propagation in the cerebrum and their pharmacological reactivity. Because such recordings are precluded for obvious ethical and technical reasons, non-invasive functional neuroimaging techniques represent a suitable approach to unravel the existence of PGO waves in humans. Here, using positron emission tomography and cerebral blood flow determination, we have shown that the lateral geniculate bodies and the occipital cortex are more closely related to rapid eye movements during PS than during wakefulness, suggesting that mechanisms similar to PGO waves are indeed in action during PS in humans. The potential relationships between the presence of PGO waves and the mechanisms involved or triggered by brain plasticity during sleep in humans are of outstanding interest and require further investigations. 


\section{REFERENCES}

Amzica, F., Neckelmann, D., and Steriade, M. 1997. Instrumental conditioning of fast (20- to 50- Hz) oscillations in corticothalamic networks. Proc. Natl. Acad. Sci. USA 94:19851989.

Amzica, F., and Steriade, M. 1996. Progressive cortical synchronization of ponto-geniculooccipital potentials during rapid eye movement. Neuroscience 72:309-314.

Aserinsky, E., Lynch, J. A., Mack, M. E., Tzankoff, S. P., and Hurn, E. 1985. Comparison of eye motion in wakefulness and REM sleep. Psychophysiology 22:1-10.

Bodis, W., Bucher, S. F., Seelos, K. C., Paulus, W., Reiser, M., and Oertel, W. H. 1997. Functional MRI mapping of occipital and frontal cortical activity during voluntary and imagined saccades. Neurology 49:416-420.

Bowker, R. M., and Morrison, A. R. 1976. The startle reflex and PGO spikes. Brain Res. 102:185-190.

Brandt, S. A., \& Stark, L. W. 1997. Spontaneous eye movements during visual imagery reflect the content of the visual scene. J. Cogn. Neurosci. 9:27-38.

Braun, A. R., Balkin, T. J., Wesensten, N. J., Gwadry, F., Carson, R. E., Varga, M., Baldwin, P., Belenky, G., and Herscovitch, P. 1998. Dissociated pattern of activity in visual cortices and their projections during human rapid eye movement sleep. Science 279:91-95.

Callaway, C. W., Lydic, R., Baghdoyan, H. A., and Hobson, J. A. 1987. Pontogeniculooccipital waves: spontaneous visual system activity during rapid eye movement sleep. Cell. Mol. Neurobiol. 7:105-149.

Chen, W., Kato, T., Zhu, X. H., Strupp, J., Ogawa, S., and Ugurbil, K. 1998. Mapping of lateral geniculate nucleus activation during visual stimulation in human brain using fMRI. Magn. Reson. Med. 39:89-96. 
Darby, D. G., Nobre, A. C., Thangaraj, V., Edelman, R., Mesulam, M. M., and Warach, S. 1996. Cortical activation in the human brain during lateral saccades using EPISTAR functional magnetic resonance imaging. NeuroImage 3:53-62.

Datta, S. 1999a. PGO wave generation: mechanism and functional significance. In Rapid Eye Movement Sleep (B. N. Mallick, and S. Inoue, Eds.) pp. 91-106. Narosa Publishing House, New Dehli.

Datta, S. 1999b. A physiological substrate for sleep dependent memory processing. Sleep Research Online 2:23

Davenne, D., and Adrien, J. 1984. Suppression of PGO waves in the kitten: anatomical effects on the lateral geniculate nucleus. Neurosci. Lett. 45:33-38.

Dement, W. 1964. Eye movements during sleep. In The oculomotor system (M. Bender, editor). pp. 366-416. Harper and Row, New York.

Frackowiak, R., Friston, K., Frith, C., Dolan, R., and Mazziotta, J. C. 1997. Human Brain Function. Academic Press, London.

Fuchs, A. F., and Ron, S. 1968. An analysis of rapid eye movements of sleep in the monkey. Electroencephalogr. Clin. Neurophysiol. 25:244-251.

Fukuda, T., Wakawura, M., and Ishikawa, S. 1981. Comparative study of eye movements in the alert state and rapid eye movement sleep. Neuro-Ophtalmology 1:253-260.

Hobson, J. A. 1964. L'activité électrique du cortex et du thalamus au cours du sommeil désynchronisé chez le chat. C. R. Soc. Biol. (Paris) 158:2131-2135.

Hobson, J. A., and McCarley, RW. 1977. The brain as a dream generator: the activationsynthesis hypothesis of the dream process. Am. J. Psychiatr. 134:1335-1348.

Hodgson, T. L., Bajwa, A., Owen, A. M., and Kennard, C. 2000. The strategic control of gaze direction in the Tower-of-London task. J. Cogn. Neurosci. 12:894-907. 
Hong, C. C., Gillin, J. C., Dow, B. M., Wu, J., and Buchsbaum, M. S. 1995. Localized and lateralized cerebral glucose metabolism associated with eye movements during REM sleep and wakefulness: a positron emission tomography (PET) study. Sleep 18:570580.

Hong, C. C., Potkin, S. G., Antrobus, J. S., Dow, B. M., Callaghan, G. M., and Gillin, J. C. 1997. REM sleep eye movement counts correlate with visual imagery in dreaming: a pilot study. Psychophysiology 34:377-381.

Inoué, S., Saha, U. K., and Musha, T. 1999. Spatio-temporal distribution of neuronal activities and REM sleep. In Rapid Eye Movement Sleep. (B. N. Mallick, and S. Inoue, Eds.) pp. 214-220. Narosa Publishing, New Dehli.

Jeannerod, M., and Mouret, J. 1963. Recherches sur les mécanismes des mouvements des yeux observés au cours de la veille et du sommeil. Pathologie-Biologie 11:1053-1060.

Jouvet, M., Michel, F., and Courjon, J. 1959. L'activité électrique du rhinencéphale au cours du sommeil chez le chat. C. R. Soc. Biol. (Paris) 153:101-105.

Jueptner, M., and Weiller, C. 1995. Review: does measurement of regional cerebral blood flow reflect synaptic activity? Implications for PET and fMRI. NeuroImage 2:148156.

Le Bihan, D., Turner, R., Zeffiro, T. A., Cuenod, C. A., Jezzard, P., and Bonnerot, V. 1993. Activation of human primary visual cortex during visual recall: a magnetic resonance imaging study. Proc. Natl. Acad.Sci. USA 90:11802-11805.

Leigh, R. J., and Zee, D. S. 1991. The neurology of eye movements. F.A. Davis Company, Philadelphia.

Llinas, R., and Ribary, U. 1993. Coherent 40-Hz oscillation characterizes dream state in humans. Proc. Natl. Acad. Sci. USA 90:2078-81. 
Llinas, R., Ribary, U., Contreras, D., and Pedroarena, C. 1998. The neuronal basis for consciousness. Philos. Trans. R. Soc. Lond. B Biol. Sci. 353:1841-1849.

Mandai, O., Guerrien, A., Sockeel, P., Dujardin, K., and Leconte, P. 1989. REM sleep modifications following a Morse code learning session in humans. Physiol. Behav. 46:639-642.

Maquet, P., Laureys, S., Peigneux, P., Fuchs, S., Petiau, C., Phillips, C., Aerts, J., Del Fiore, G., Degueldre, C., Meulemans, T., Luxen, A., Franck, G., Van Der Linden, M., Smith, C., Cleeremans, A. 2000. Experience-dependent changes in cerebral activation during human REM sleep. Nature Neurosci. 3:831-836.

Maquet, P., Péters, J.-M., Aerts, J., Delfiore, G., Degueldre, C., Luxen, A., and Franck, G. 1996. Functional neuroanatomy of human rapid-eye-movement sleep and dreaming. Nature 383:163-166.

McCarley, R. W., Winkelman, J. W., and Duffy, F. H. 1983. Human cerebral potentials associated with REM sleep rapid eye movements: links to PGO waves and waking potentials. Brain Res. 274:359-364.

Mikiten, T. H., Niebyl, P. H., and Hendley, C. D. 1961. EEG desynchronization during behavioral sleep associated with spike discharges from the thalamus of the cat. Fed. Proc. 20:327

Mouret, J., Jeannerod, M., and Jouvet, M. 1963. L'activité électrique du système visuel au cours de la phase paradoxale du sommeil chez le chat. J. Physiol. (Paris) 55:305-306.

Nelson, J. P., McCarley, R. W., and Hobson, J. A. 1983. REM sleep burst neurons, PGO waves, and eye movement information. J. Neurophysiol. 50:784-97.

Paus, T., Marrett, S., Worsley, K. J., and Evans, A. C. 1995. Extraretinal modulation of cerebral blood flow in the human visual cortex: implications for saccadic suppression. J. Neurophysiol. 74:2179-83. 
Paus, T., Petrides, M., Evans, A. C., and Meyer, E. 1993 Aug. Role of the human anterior cingulate cortex in the control of oculomotor, manual, and speech responses: a positron emission tomography study. J. Neurophysiol. 70:453-469.

Petit, L., Orssaud, C., Tzourio, N., Salamon, G., Mazoyer, B., and Berthoz, A. 1993. PET study of voluntary saccadic eye movements in humans: basal ganglia-thalamocortical system and cingulate cortex involvement. J. Neurophysiol. 69:1009-1017.

Rechtschaffen, A., and Kales, A. A. 1968. A manual of standardized terminology, techniques and scoring system for sleep stages of human sujects. US Department of Health, Education and Welfare, Bethesda.

Salzarulo, P., Lairy, G. C., Bancaud, J., and Munari, C. 1975. Direct depth recording of the striate cortex during REM sleep in man: are there PGO potentials? EEG Clin. Neurophysiol. 38:199-202.

Schlag, J., and Schlag-Rey, M. 1987. Evidence for a supplementary eye field. J. Neurophysiol. 57:179-200.

Shaffery, J. P., Roffwarg, H. P., Speciale, S. G., and Marks, G. A. 1999. Ponto-geniculooccipital-wave suppression amplifies lateral geniculate nucleus cell-size changes in monocularly deprived kittens. Brain Res. Dev. Brain Res. 114:109-119.

Smith, C., and Lapp, L. 1991. Increases in number of REMs and REM density in humans following an intensive learning period. Sleep 14:325-330.

Talairach, J., and Tournoux, P. 1988. Co-Planar Stereotaxic Atlas of the Human Brain. Thieme, New York.

Vanni-Mercier, G., Pelisson, D., Goffart, L., Sakai, K., and Jouvet, M. 1994. Eye saccade dynamics during paradoxical sleep in the cat. Eur. J. Neurosci. 6:1298-1306.

Vanni-Mercier, G., and Debilly, G. 1998. A key role for the caudoventral pontine tegmentum in the simultaneous generation of eye saccades in bursts and associated ponto- 
geniculo-occipital waves during paradoxical sleep in the cat. Neuroscience 86:571585.

Vuillon-Cacciuttolo, G., and Seri, B. 1978. Effects of optic nerve section in baboons on the geniculate and cortical spike activity during various states of vigilance. Electroencephalogr. Clin. Neurophysiol. 44:754-768.

\section{ACKNOWLEDGEMENTS}

This work was supported by the Fonds National de la Recherche Scientifique de Belgique (FNRS), the Fondation Médicale Reine Elisabeth (FMRE), the Research Fund of ULg, and the PAI/IAP Interuniversity Pole of Attraction P4/22. PM is FNRS Senior Research Associate. SL is FNRS Postdoctoral Researcher. PP is supported by PAI. Personal thanks are expressed to C. Frith for helpful comments on a previous version of this manuscript, P. Hawotte and J.-L. Genon for technical assistance. 
TABLE 1. Brain areas where rCBF correlated with spontaneous ocular movements (REMs counts) during wakefulness.

\begin{tabular}{|c|c|c|c|c|c|}
\hline Side & Region & $\mathrm{x}$ & $\mathrm{y}$ & $\mathrm{Z}$ & $\mathrm{T}$ \\
\hline Left & Medial Frontal Gyrus (BA 10) & -6 & 68 & -4 & 4.68 \\
\hline Left & Superior Frontal Gyrus (BA 11) & -8 & 70 & -12 & 4.35 \\
\hline Left & Medial Frontal Gyrus (BA 9) & -16 & 42 & 24 & 4.02 \\
\hline Left & Superior Frontal Gyrus (BA 9) & -16 & 38 & 34 & 3.49 \\
\hline Right & Postcentral Gyrus (BA 5) & 10 & -48 & 68 & 3.79 \\
\hline Left & Inferior Frontal Gyrus (BA 46) & -58 & 30 & 8 & 3.64 \\
\hline Right & Orbital Gyrus (BA 11) & 16 & 38 & -30 & 3.57 \\
\hline
\end{tabular}

Note. $\mathrm{x}, \mathrm{y}, \mathrm{z}$ are coordinates $(\mathrm{mm})$ in the standard Talairach and Tournoux (1988) stereotactic space. $\mathrm{BA}=$ Brodmann area. $\mathrm{T}=\mathrm{t}$-statistic value. All values are significant at $\mathrm{p}<.001$ (uncorrected). 
TABLE 2. Brain areas where rCBF correlated with REMs counts during PS.

\begin{tabular}{lllllll} 
Side & Region & $\mathrm{x}$ & $\mathrm{y}$ & $\mathrm{z}$ & $\mathrm{T}$ & $\mathrm{p}^{\mathrm{corr}}$ \\
\hline Right & Occipital cortex (BA 17) & 12 & -84 & 4 & 4.55 & 0.000 \\
Right & Anterior cingulate cortex (BA 32) & 10 & 2 & 44 & 4.54 & 0.000 \\
Right & Medial frontal gyrus [Supplementary & 18 & 2 & 50 & 4.53 & 0.000 \\
& motor area (BA 6)] & & & & & \\
Right & Parahippocampal gyrus (BA 35) & 18 & -28 & -6 & 4.46 & 0.001 \\
Right & Geniculate body & 16 & -28 & -2 & 4.27 & 0.000 \\
Right & Thalamus & 2 & -8 & 8 & 3.41 & 0.005 \\
Right & Precuneus (BA 7) & 6 & -42 & 50 & 3.44 & 0.007 \\
Left & Mesencephalon & -10 & -36 & -10 & 3.39 & 0.007
\end{tabular}

Note. $x, y, z$ are coordinates (mm) in the standard Talairach and Tournoux (1988) stereotactic space. $\mathrm{BA}=$ Brodmann area. $\mathrm{T}=\mathrm{t}$-statistic value. $\mathrm{p}^{\text {corr }}=\mathrm{p}$ corrected for multiple comparisons in a small volume $(5 \mathrm{~mm})$. 
TABLE 3. Brain areas more activated in relation to REMs counts during PS than during wakefulness [condition (PS versus Wakefulness) by performance (REMs counts) interaction].

\begin{tabular}{lllllll} 
Side & Region & $\mathrm{x}$ & $\mathrm{y}$ & $\mathrm{z}$ & $\mathrm{T}$ & $\mathrm{p}^{\text {corr }}$ \\
\hline Right & Occipital cortex (BA 17) & 20 & -86 & -6 & 4.60 & 0.000 \\
Left & Occipital cortex (BA 17) & -10 & -92 & -6 & 4.00 & 0.001 \\
Right & Geniculate body & 16 & -28 & -2 & 3.94 & 0.001
\end{tabular}

Not included in the a priori hypothesis

$\begin{array}{llllll}\text { Right } & \text { Sensorimotor cortex (BA 4) } & 46 & -20 & 40 & 3.60 \\ \text { Right } & \text { Parahippocampal gyrus (BA 35) } & 20 & -30 & -8 & 4.41 \\ \text { Right } & \text { Premotor cortex (BA 6) } & 62 & 4 & 46 & 3.51 \\ \text { Left } & \text { Anterior cingulate cortex (BA 24) } & -6 & -2 & 36 & 3.68\end{array}$

Note. $x, y, z$ are coordinates (mm) in the standard Talairach and Tournoux (1988) stereotactic space. $\mathrm{BA}=$ Brodmann area. $\mathrm{T}=\mathrm{t}$-statistic value. $\mathrm{p}^{\text {corr }}=\mathrm{p}$ corrected for multiple comparisons in a small volume $(5 \mathrm{~mm})$. 


\section{FIGURE CAPTION}

Cerebral areas more active in relation to REMs during PS than during wakefulness. Upper left panel: sagittal section $16 \mathrm{~mm}$ to the right of the midline. Right panel: transverse sections from $-4 \mathrm{~mm}$ to $0 \mathrm{~mm}$ from the bi-commissural plane. The functional data are displayed at $\mathrm{p}<$ 0.001 uncorrected, superimposed on the average MRI of the subjects, coregistered to the same reference space. Left bottom panel: design matrix. Middle bottom panel: plot of the regional adjusted CBF (arbitrary units) in the right geniculate body in relation to the rapid eye movement counts. The geniculate $\mathrm{CBF}$ is correlated to the rapid eye movement counts more during PS (in red) than during wakefulness (in green). 

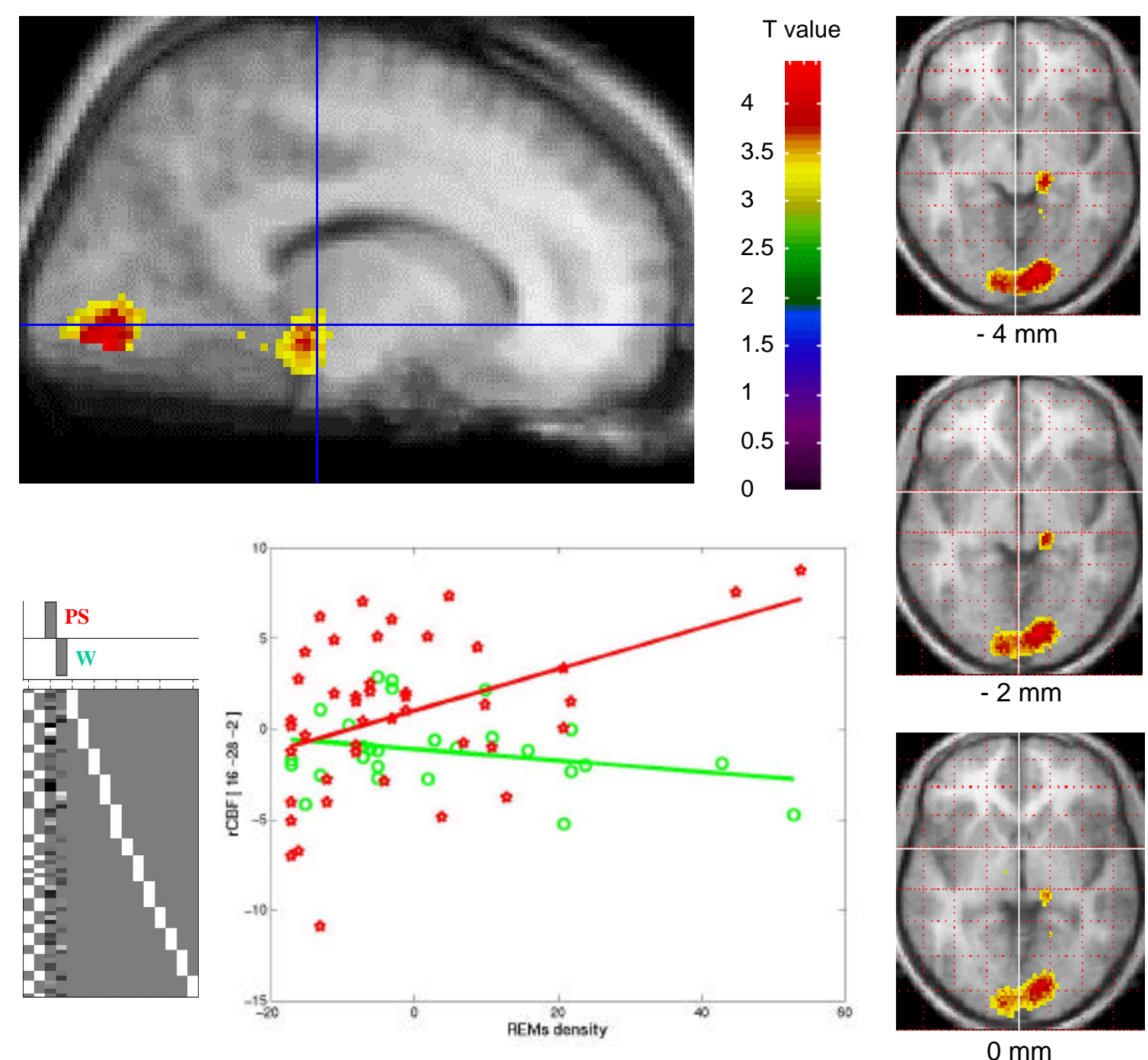